\title{
Viscosity-temperature relationship of glasses with low melting points obtained by wide-range measurement
}

\author{
Hiroshi KOBAYASHI ${ }^{\dagger}$ and Haruyuki TAKAHASHI* \\ National Metrology Institute of Japan, AIST, Retired, 8-1-309, 2-chome, Tomooka, Nagaokakyo 617-0843 \\ *Graduate School of Science and Engineering, Ibaraki University, 4-12-1, Nakanarusawa, Hitachi 316-8511
}

The viscosity-temperature relationships for metaphosphate glass and polystyrene in the wide-temperature range, including below the glass transition temperature $T_{\mathrm{g}}$, obtained by us, were analyzed using the Adam-Gibbs theory. The results showed that the glass transition is a non-equilibrium phase transition from the Vogel-Tammann-Fulcher (VTF) state to the Arrhenius state occurring at $T_{\mathrm{g}}$. The Arrhenius curves below $T_{\mathrm{g}}$ show different slopes with varying activation energies owing to the fictive temperatures $\boldsymbol{T}_{\mathrm{f}}$ characterizing the structural states of the glasses. To explain this result from the microscopic viewpoint, a model of an intermediate range order (IRO) is proposed. We also propose that the glass transition is due to a self-organization of the IROs, a few nanometers in size, in the glass state.

(02008 The Ceramic Society of Japan. All rights reserved.

Key-words: Glass transition, Viscosity, Metaphosphate glass, Polystyrene, Adam-Gibbs theory, Intermediate range order, Icosahedron, Self-organization

[Received March 21, 2008; Accepted May 15, 2008]

\section{Introduction}

Glass-forming materials show large temperature changes in volume and enthalpy, particularly near $T_{\mathrm{g}}$. The change in the volume results in a jump in thermal expansion, while that in the enthalpy results in a jump in heat capacity. Aside from these changes, the temperature dependence of the viscosities of such materials shows a much larger change of about 10 orders of magnitude near $T_{\mathrm{g}}$. Viscosity is a dynamic correlation in materials, which is an important property of the complex system of increasing interest in recent physics. It is difficult to measure viscosity below $T_{\mathrm{g}}$. Thus, viscosity measurements in the wide temperature range even below $T_{\mathrm{g}}$ are strongly sought in order to explain the glass transition mechanism. The Adam-Gibbs theory ${ }^{1)}$ clearly describes the viscosity-temperature relationship for glass-forming materials, which provides a fragile-strong classification of these materials by evaluating their configurational entropies.

\section{Experimental procedure}

We have reported the temperature dependencies of the viscosities of metaphosphate glasses, ${ }^{2)}$ namely, $\mathrm{AgPO}_{3}$ and $(\mathrm{AgI})_{0.5}\left(\mathrm{AgPO}_{3}\right)_{0.5}$, and polystyrene with low melting points in the liquid and glass states obtained by the method that we developed. ${ }^{3)}$ The results show the VTF Eq. in the viscosity-temperature relationship above $T_{\mathrm{g}}$ and the Arrhenius Eq. below $T_{\mathrm{g}}$. In this study, the results obtained are analyzed using the Adam-Gibbs theory by evaluating the configurational entropy on introducing $T_{\mathrm{f}} . T_{\mathrm{f}}$ describes the structural state of the glass. In addition, a model of the IRO is produced in order to explain the results from the microscopic viewpoint.

In Fig. 1, the temperature dependence of the viscosity of metaphosphate glass reported in our paper ${ }^{2}$ is shown. The data marked $(\mathrm{O})$ in the low-temperature region was obtained using

\footnotetext{
Corresponding author: H. Kobayashi; E-mail: hkoba@mx2.canvas. ne.jp
}

our method, which is described in detail in the related paper. ${ }^{3)}$ The data marked $(\Delta)$ in the high-temperature region was obtained by a commercial oscillating disk method. These two types of data continue smoothly near $10^{8} \mathrm{~Pa} \cdot \mathrm{s}$ and show two temperature Eqs. as follows.

In the temperature region of $T>T_{\mathrm{g}}$, we obtain the VTF Eq.

$$
\log \eta=\log \eta_{0}+\frac{B}{\left(T-T_{0}\right)},
$$

where $\eta$ is the viscosity, $T$ is the temperature, and $\eta_{0}, B$ and $T_{0}$ are constants. We obtain $\log \eta_{0}=-2$ in Eq. (1). In the tempera-

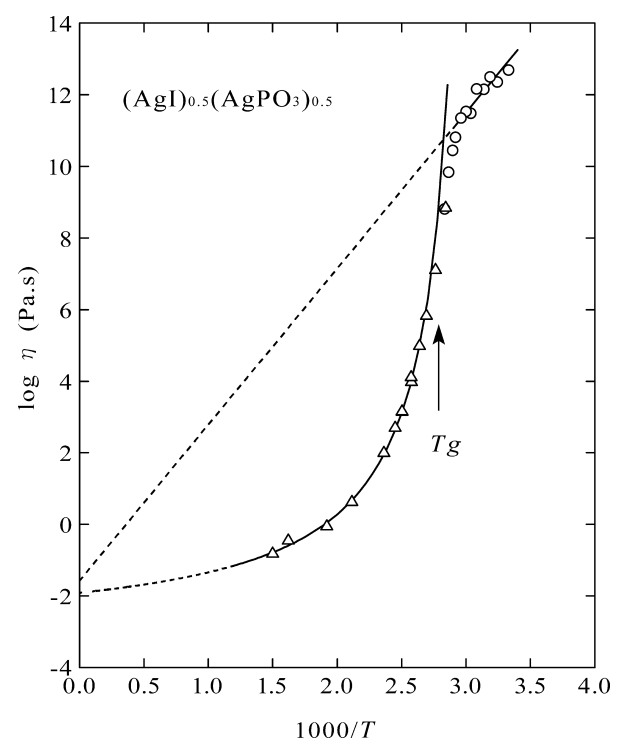

Fig. 1. Temperature dependence of the viscosity $\eta$ of metaphosphate glass $(\mathrm{AgI})_{0.5}\left(\mathrm{AgPO}_{3}\right)_{0.5}$. Data $(\mathrm{O})$ was obtained using our method, while data $(\Delta)$ was obtained using an oscillating disk viscometer. 


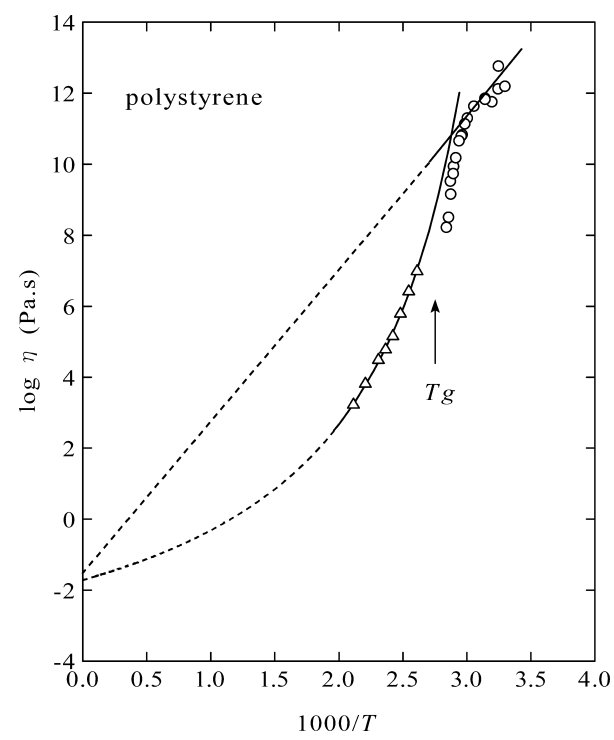

Fig. 2. Temperature dependence of the viscosity $\eta$ of polystyrene. Data $(\mathrm{O})$ was obtained using our method, while data $(\Delta)$ was obtained using an oscillating disk viscometer.

ture region of $T<T_{\mathrm{g}}$, we obtain the Arrhenius Eq.

$$
\log \eta=\log \eta_{0}+\frac{E}{R T},
$$

where $E$ is the activation energy and $R$ is the gas constant. We obtain $\log \eta_{0}=-2$ in Eq. (2). Note that the VTF and Arrhenius curves converge to the same value at $1 / T=0$. This suggests that there are two states, namely, the VTF state in liquid and the Arrhenius state in glass, and that a phase transition from the VTF state to the Arrhenius state at $T_{\mathrm{g}}$ is expected.

In Fig. 2, the temperature dependence of the viscosity of polystyrene cited from Ref. 2 is shown; we can see the same result for metaphosphate glass, as shown in Fig. 1. We obtain $\log \eta_{0}=-2$ in Eq. (1) $\left(T>T_{\mathrm{g}}\right)$ and $\log \eta_{0}=-2$ in Eq. (2) $(T<$ $T_{\mathrm{g}}$ ) for polystyrene.

Plazek and Magill ${ }^{4}$ measured the viscosity of aromatic hydrocarbon over a wide temperature range and obtained the same Eqs. as those observed in metaphosphate glass and polystyrene. From their data, we obtained $\log \eta_{0}=-3$ in Eq. (1) $\left(T>T_{\mathrm{g}}\right)$ and $\log \eta_{0}$ $=-3$ in Eq. (2) $\left(T<T_{\mathrm{g}}\right)$ for aromatic hydrocarbon. Angell ${ }^{5), 6)}$ measured the viscosities of $\mathrm{ZrF}_{4}$-based glass and $\mathrm{BeF}_{2}$-based glass at $T>T_{\mathrm{g}}$. He obtained $\log \eta_{0}=-5$ for $\mathrm{ZrF}_{4}$-based glass and $\log \eta_{0}$ $=-5$ for $\mathrm{BeF}_{2}$-based glass in Eq. (1) $\left(T>T_{\mathrm{g}}\right)$.

We measured the viscosities of as-received, annealed, and loaded polystyrenes, ${ }^{2)}$ which have different structures, with $T_{\mathrm{f}}$ characterizing the structural states, in the low temperature range. The data is shown in Fig. 3. The data marked $(\mathrm{O})$ was obtained for the as-received specimen, the data marked $(\Delta)$ was obtained for the specimen annealed for $24 \mathrm{~h}$ at $70^{\circ} \mathrm{C}$, and the data marked $(\nabla)$ was obtained for the specimen kept for one week under a load of $9.8 \mathrm{~N}(1 \mathrm{~kg})$. The temperature dependencies of the viscosities of polystyrenes shown in Fig. 3 show the same curve at $T_{\mathrm{g}} / T<1.08$, but show different slopes at $T_{\mathrm{g}} / T>1.08$ owing to the treatments that result in the different structural states, which are characterized by $T_{\mathrm{f}}$. The slopes of the viscosities for the three specimens with different values of $T_{\mathrm{f}}$ converge at a viscosity of $10^{11} \mathrm{~Pa} \cdot \mathrm{s}$ near $T_{\mathrm{g}} / T=1$. This data means that the viscosity of the as-received specimen shows a value of $10^{-2} \mathrm{~Pa} \cdot \mathrm{s}$ at $1 / T=0$ but

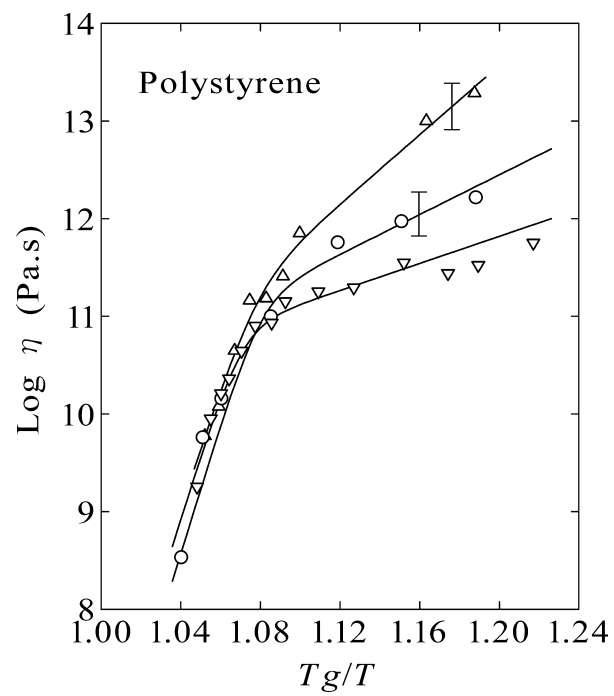

Fig. 3. Temperature dependence of the viscosity $\eta$ of polystyrene. Data $(\mathrm{O})$ was obtained for the as-received specimen, data $(\Delta)$ was obtained for the specimen annealed for $24 \mathrm{~h}$ at $70^{\circ} \mathrm{C}$, and data $(\nabla)$ was obtained for the specimen kept for one week under a load of $9.8 \mathrm{~N}$.

those of the annealed and loaded specimens do not show the value of $10^{-2} \mathrm{~Pa} \cdot \mathrm{s}$ at $1 / T=0$. It is supposed that the former specimen shows the real phase in the Arrhenius behavior, but the latter ones do not show such phase because of their additional treatments. If the three curves have the real phase in the Arrhenius behavior, these curves are expected to converge at the same value of $10^{-2} \mathrm{~Pa} \cdot \mathrm{s}$ at $1 / T=0$. The viscosity of $10^{-2} \mathrm{~Pa} \cdot \mathrm{s}$ at $1 / T=0$ represents a virtual value, which was not realized in the experiment.

As results, the fragile glasses ${ }^{7}$ show the temperature dependencies of viscosities which display a VTF Eq. at $T>T_{\mathrm{g}}$ and an Arrhenius one at $T<T_{\mathrm{g}}$, and the Arrhenius curves show different slopes in different non-equilibrium states.

Ryu and Tomozawa ${ }^{8)}$ reported the Arrhenius-type dependencies of the viscosities of silica glasses in the low-temperature range with different $T_{\mathrm{f}}$ values, which show different slopes. They made no mention that these Arrhenius curves for silica glass converge to the same value at $1 / T=0$.

Following the Adam-Gibbs theory, the viscosity $\eta$ of a glassforming material is shown as

$$
\eta=\eta_{0} \exp \left[\frac{B}{T S_{\mathrm{c}}}\right]
$$

where $\eta_{0}$ and $B$ are constants, and $S_{\mathrm{c}}$ is the configurational entropy. At $T>T_{\mathrm{g}}$,

$$
S_{\mathrm{c}}=\Delta C_{\mathrm{p}} \frac{\left(T-T_{0}\right)}{T},
$$

where $\Delta C_{\mathrm{p}}$ is the configurational heat capacity. Here, $\mathrm{Sc}$ is not constant. This means that the configuration of molecules changes with temperature and that the material is present in a state of equilibrium. The material is called a supercooled liquid, which is in a meta-stable state. As results, we obtain the VTF Eq.

$$
\eta=\eta_{0} \exp \left[\frac{D}{\left(T-T_{0}\right)}\right],
$$

where $\mathrm{D}$ is a constant

$$
\text { At } T<T_{\mathrm{g}} \text {, }
$$




$$
S_{\mathrm{c}}=\Delta C_{\mathrm{p}} \frac{\left(T_{\mathrm{f}}-T_{0}\right)}{T_{\mathrm{f}}},
$$

where $\Delta C_{\mathrm{p}}$ is constant. Here, $S_{\mathrm{c}}$ is constant. This means that the configuration of molecules freezes into a glass state, which is a non-equilibrium state characterized by $T_{\mathrm{f}}$. As results, we obtain the Arrhenius Eq.

$$
\begin{aligned}
& \eta=\eta_{0} \exp \left[\frac{E}{R T}\right], \\
& E \propto \frac{T_{\mathrm{f}}}{\left(T_{\mathrm{f}}-T_{0}\right)}=\frac{1}{1-\left(T_{0} / T_{\mathrm{f}}\right)} .
\end{aligned}
$$

In Eq. (8), $\mathrm{E}$ is larger when $T_{\mathrm{f}}$ is smaller, which means a more equilibrial state. In Fig. 3 , it is presumed that the $T_{\mathrm{f}}$ of the annealed specimen is smaller than that of the as-received specimen, and that the $T_{\mathrm{f}}$ of the loaded specimen is larger than that of the as-received specimen. This means that the annealed specimen is present in the more equilibrial state than the asreceived specimen, and that the loaded specimen is in the less equilibrial state than the as-received specimen.

\section{Results and discussion}

The results are shown in Fig. 4 in which the VTF and Arrhenius curves converge to the same value at $1 / T=0$ and Arrhenius curves have different slopes with different $\mathrm{E}$ values at $T<T_{\mathrm{g}}$. In Fig. 4, $T_{\mathrm{f} 0}, T_{\mathrm{f} 1}$, and $T_{\mathrm{f} 2}\left(T_{\mathrm{f} 0}<T_{\mathrm{f} 1}<T_{\mathrm{f} 2}\right)$ are the fictive temperatures representing the structural states of the glasses. Figure 4 clearly reproduces the experimental results that we obtained. It is desired that these Eqs. are solved by a basic theory.

In the next section, we propose a model of the IRO for explaining the results from the microscopic viewpoint. The potential energy of a glass-forming material is given as a function of atomic coordinates, which occupy the minimum sites in the potential energy surface (energy landscape). ${ }^{9-11)}$ The portion of the potential energy surface representing the liquid $\left(T>T_{\mathrm{g}}\right)$

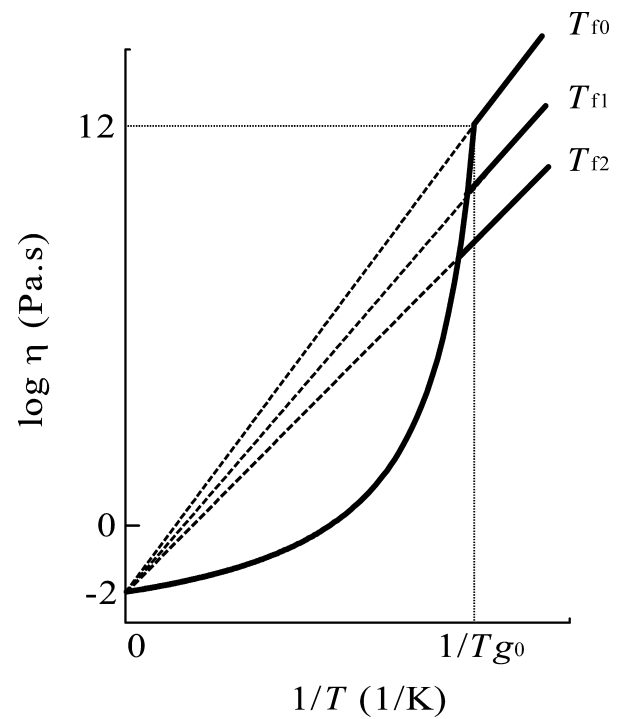

Fig. 4. Temperature dependence of the viscosities $\eta$ of fragile glasses estimated by the Adam-Gibbs theory. $T_{\mathrm{f} 0}, T_{\mathrm{f} 1}$, and $T_{\mathrm{f} 2}\left(T_{\mathrm{f} 0}<T_{\mathrm{f} 1}<T_{\mathrm{f} 2}\right)$ show the fictive temperatures representing the structural states of the glasses. and glass $\left(T<T_{\mathrm{g}}\right)$ regions has a large minima with varying depth. It is expected that the VTF state $\left(T>T_{\mathrm{g}}\right)$ has a potential distribution with larger traps in the energy surface than the Arrhenius state $\left(T<T_{\mathrm{g}}\right)$, because the effective activation energy $(\mathrm{d} \log \eta / \mathrm{d} 1 /$ $T$ ) of the VTF state is higher than that of the Arrhenius state. This is in accordance with our interpretation that the energy landscape for fragile glass (VTF-type) is more heterogeneous than that for strong glass (Arrhenius-type) ${ }^{11)}$ Figure 5 shows the double-well energy potential of a glass-forming material when external stress is applied. The double-well potential is a different expression of the energy landscape of this material. Through a thermal activation process, the occupied energy states of glass or liquid surmount the barrier height $U$ from one potential minimum to another after stress application. This leads to the thermodynamic understanding of the viscous process in glass and liquid under external stress. $U$ is proportional to activation energy in the thermal activation process. The constant activation energy of the glass state $\left(T<T_{\mathrm{g}}\right)$ indicates the freezing of the molecule configurations constituted by the IROs, because their energy surface freezes in spite of the temperature changes. The IRO is a transient configuration of molecules that changes in size and number with time and temperature. It is presumed that the size and number of IROs are not uniformly distributed through glass owing to the local rearrangements of molecules in liquid. Sometimes, the IRO is called a cooperatively rearranging region (CRR), ${ }^{1)}$ spatial heterogeneity, ${ }^{12)}$ and cluster, ${ }^{13)}$ which are strongly related to the static and dynamic properties of glasses as well as to the glass transition.

It is well known that there are short-range orders of about 2 A in liquids, glasses, and crystals. Recently, the presence of medium-range structures in supercooled liquids and glasses has been reported as icosahedrons in metallic glasses. ${ }^{14)}$ Jonsson and Andersen ${ }^{15)}$ have previously reported that the icosahedral ordering seen in molecular-dynamic simulations of supercooled liquid and glass is strongly related to the glass transition phenomena. Their size is estimated to be about 5-10 A. We presume that these are precursors of the IROs. Fukunaga et al. ${ }^{16)}$ performed a topological characterization of $\mathrm{NiZr}_{2}$ and $\mathrm{CuZr}_{2}$ metallic glasses by the neutron/X-ray diffraction method, and reported that the icosahedrons confirmed by them play an important role in stabilizing the metallic glass configuration. Hirotsu et al. ${ }^{17)}$ reported that the IROs in PdNiP bulk metallic glass are crystalline nanoclusters of about $2 \mathrm{~nm}$ in size, as determined by transmission electron microscopy. They reported that a very small interfacial energy between the nanophase and the matrix can form a meta-stable amorphous phase with structural fluctuation. Russell and Israeloff $^{18)}$ reported the lifetime of heterogeneities or cooperativities in polyvinylacetate by measuring their local dielectric fluc-

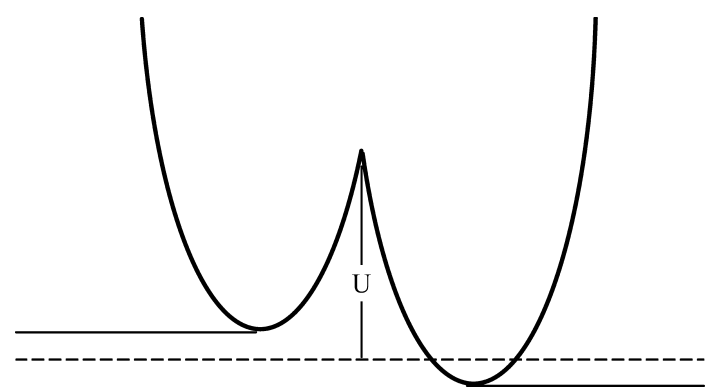

Fig. 5. Double-well energy potential of liquid and glass under external stress. U represents the barrier height in the energy potential. 
tuations near $T_{\mathrm{g}}$ using atomic force microscopy. They reported that the lifetime of cooperativities obtained is comparable to that of the $\alpha$-relaxation (stress relaxation) and that the cooperativity takes the form of transient molecular clusters a few nanometers in size. This result means that there is the same microscopic mechanism in the cooperative rearrangement of molecules and the $\alpha$-relaxation. These observations confirm the IRO model. In general, the stress relaxation time obtained by mechanical recovery is smaller by an order of magnitude than the structural relaxation time obtained by thermal recovery. ${ }^{19), 20)}$

Tanaka ${ }^{21,22)}$ reported interesting simulations related to the liquid-liquid transition using a two-order parameter model. $\mathrm{He}$ proposed a transition from the ordinary liquid state to the frustrated meta-stable liquid state at $T_{\mathrm{m}}{ }^{*}$, which is characterized by the appearance of meta-stable solid-like islands (medium-range crystalline orders or IROs) and the resulting appearance of the cooperative nature of $\alpha$ relaxation. The former liquid shows the Arrhenius behavior and the latter shows the VTF behavior. He made no mention about the transition mechanism from a VTF state to an Arrhenius state occurring at $T_{\mathrm{g}}$. Tanaka and colleagues $^{23), 24)}$ have previously found that this new transition obtained by the simulation is a first order phase transition in the experiment of triphenyl phosphate. These results indicate that the IROs are very important for studying the cooperative phenomena, including the glass transition.

\section{Conclusion}

In conclusion, fragile glasses experimentally and theoretically show temperature dependencies for their viscosities of a VTF Eq. at $T>T_{\mathrm{g}}$ and an Arrhenius Eq. at $T<T_{\mathrm{g}}$, and Arrhenius Eqs. show different slopes in different non-equilibrium states characterized by $T_{\mathrm{f}}$. We presume that the IROs, which increase in size and number through rearrangements with decreasing temperature down to $T_{\mathrm{g}}$, suppress the crystallization of liquids and generate glasses through freezing below $T_{\mathrm{g}}$. It was confirmed that the IROs in metallic glasses are crystalline nanoclusters of about 2 $\mathrm{nm}$ in size and that their precursors are icosahedrons. We consider that the glass transition is due to a self-organization of the IROs in non-uniform distribution in glass-forming materials. To estimate the properties of the glasses, the sizes and boundaries of the IROs are important because their size is very small; that is, in the order of a few nanometers.

It is presumed that the glass transition is a kind of second order phase transition to a non-equilibrium state with no latent heat. It is expected that its mechanism could be solved by the renormalization group theory, which can treat complex and non- equilibrium phenomena by considering local dynamic correlations.

Acknowledgments The authors are very grateful to Professor M. Tomozawa of Rensselaer Polytechnic Institute and to Professor H. Mizubayashi of Tsukuba University for the many helpful discussions and their support.

\section{References}

1) G. Adam and J. H. Gibbs, J. Chem. Phys., 43, 139-146 (1965).

2) H. Kobayashi, H. Takahashi and Y. Hiki, Mater. Sci. Eng., A 442, 263-267 (2006).

3) H. Kobayashi, I. Kodama and T. Nashima, Jpn. J. Appl. Phys., 46, 7959-7962 (2007).

4) D. J. Plazek and J. H. Magill, J. Chem. Phys., 49, 3678-3682 (1968).

5) C. A. Angell, J. Non-Cryst. Solid, 131-133, 13-31 (1991).

6) C. A. Angell, "Relaxation in Complex Systems", Ed. by K. L. Ngai and G. B. Wright, National Technical Center, U. S. Department of Commerce, Springfield, VA (1992) pp. 3-11.

7) C. A. Angell, J. Phys. Chem. Solids, 49, 863-871 (1988).

8) S. R. Ryu and M. Tomozawa, J. Am. Ceram. Soc., 89, 81-88 (2006).

9) M. Goldstein, J. Chem. Phys., 51, 3728-3739 (1969).

10) F. H. Stillinger, Science, 267, 1935-1939 (1995).

11) P. G. Debenedetti and F. G. Stillinger, Nature, 410, 259-267 (2001).

12) O. Yamamuro, I. Tsukushi, A. Lindqvist, S. Takahara, M. Ishikawa and T. Matsuo, J. Phys. Chem. B, 102, 1605-1609 (1998).

13) M. Oguni, J. Non-Cryst. Solids, 210, 171-177 (1997).

14) D. W. Qi and S. Wang, Phys. Rev. B, 44, 884-887 (1991).

15) H. Jonsson and H. C. Andersen, Phys. Rev. Lett., 60, 22952298 (1988).

16) T. Fukunaga, K. Itoh, T. Otomo, K. Mori, M. Sugiyama, H. Kato, M. Hasegawa, A. Hirata, Y. Hirotsu and K. Aoki, Physica B, 385-386, 259-262 (2006).

17) Y. Hirotsu, T. G. Nieh, A. Hirata, T. Ohkubo and N. Tanaka, Phys. Rev. B, 73, 012205-1-012205-4 (2006).

18) E. V. Russell and N. E. Israeloff, Nature, 408, 695-698 (2000).

19) S. M. Rekhson and O. V. Mazurin, J. Am. Ceram. Soc., 57, 327-328 (1974).

20) M. M. Santore, R. S. Duran and G. B. McKenna, Polymer, 32, 2377-2381 (1991).

21) H. Tanaka, J. Non-Cryst. Solids, 351, 3385-3395 (2005).

22) H. Shintani and H. Tanaka, Nature Phys., 2, 200-206 (2006).

23) H. Tanaka, R. Kurita and H. Mataki, Phys. Rev. Lett., 92, 025701-1-025701-4 (2004).

24) R. Kurita and H. Tanaka, Science, 306, 845-848 (2004). 